\title{
Investigation of Lithium Sorption Efficiency Using SWCNT Functionalized Electrospun Fiber Mats from the Hypersaline Geothermal Brine
}

\author{
Aslı ÇELIK ${ }^{1, a}$, Gökhan TOPÇU ${ }^{1, b}$, Tuğba ISIK ${ }^{1, c}$, Alper BABA ${ }^{2, d}$, \\ Nesrin HORZUM ${ }^{3, \mathrm{e} *}$, Mustafa M. DEMIR ${ }^{1, f_{*}}$
}

İzmir Institute of Technology, ${ }^{1}$ Department of Materials Science and Engineering,

${ }^{2}$ Department of Civil Engineering, Turkey, İzmir, Urla

${ }^{3}$ İzmir Katip Çelebi University, Engineering Sciences Department, Turkey, İzmir, Çiğli

acelikasli86@gmail.com, bgokhantopcu@iyte.edu.tr, 'tugbaisik@iyte.edu.tr,

dalperbaba@iyte.edu.tr, ${ }^{e}$ nesrin.horzum.polat@ikc.edu.tr, ${ }^{f}$ mdemir@iyte.edu.tr

Keywords: Electrospinning, carbon nanotube, fibrous sorbent, geothermal mining

\begin{abstract}
Geothermal mining from brines becomes increasingly important with the increasing demand for rare earth elements in various engineering applications. Geothermal fluids contain valuable minerals and metals such as silica, zinc, lithium, and other materials that can be processed to recover these products. Solution mining by nature is challenging because of variable composition as well as the concentration of the interfering ions, particularly calcium and magnesium, and the presence of interfering ions increases the recovery costs requiring additional steps. The aim of this study is the fabrication of single-walled carbon nanotube functionalized electrospun chitosan, poly(methyl methacrylate) (PMMA), and polyacrylonitrile (PAN) fiber mats. Effect of polymer type, dilution factor, and surface modification on the sorption of lithium $\left(\mathrm{Li}^{+}\right)$ions was investigated. The maximum sorption performance was obtained with SWCNT functionalized PAN (15 wt\%) fiber mats and they have sorption percentage as $55 \%$ at diluted $(1 / 100)$ brine samples.
\end{abstract}

\section{Introduction}

Lithium is one of the rare elements and crucial due to its important applications in rechargeable batteries, medicine, light aircraft alloys and electric vehicles [1]. It is mainly acquired from two major sources: lithium mineral ores and lithium water resources including brines and seawater. Due to lower cost and more available reserves, recovery of lithium from water sources is preferable rather than lithium mineral ores [2]. Geothermal brines are utilized for energy production in Tuzla Geothermal Field (TGF), which located in northwestern Turkey, $80 \mathrm{~km}$ south of the city of Çanakkale and $5 \mathrm{~km}$ from the Aegean Sea. Lithium ion concentration in the TGF is around $28 \mathrm{ppm}$ [3]. There are several methods for the separation of cations from the aqueous systems such as ion exchange, chemical precipitation, reverse osmosis, and coagulation. However, these methods are both time-consuming and non-economic. Adsorption method is the most convenient one due to being efficient, practical, and economic. Thus, the separation of lithium or any other valuable ions for instance strontium ions in the presence of more abundant cations like $\mathrm{Na}^{+}, \mathrm{K}^{+}, \mathrm{Mg}^{2+}$, and $\mathrm{Ca}^{2+}$ in seawater or brines could be promising for mining [4]. Activated carbon, resins, hydrogels, aluminum oxides, silicates, zeolites and fly ash have been used as adsorbent materials for many years.

Electrospinning appears to be a potential method to fabricate sorbent materials due to their large surface area to volume ratio and mechanical integrity. It is a technique for the fabrication of nanofibers with diameters down to a few nanometers by applying a high potential difference to a polymer solution or melt. Although many synthetic and natural polymeric materials could have been electrospun into nanofibers, the capability of electrospinning has been successfully extended to fabricate nanofibers from polymer alloys, and polymers loaded with nanoparticles, as well as to metals and ceramics [5]. In a typical electrospinning set up, there are three basic components: a high voltage supplier, a capillary tube with a pipette or needle of small diameter, and a metal collecting 
screen. During the process, the polymer solution or melt is pumped through the capillary tube and a high voltage is used to create an electrically charged jet of polymer out of the tube. The nozzle of the capillary tube serves as a positive electrode and an electric field exists between a nozzle and grounded collector, which acts as a negative electrode. The polymer solution evaporates and solidifies through the electric field. Then, interconnected web of fibers is formed on the collecting screen [6]. The morphology of electrospun nanofibers could readily diversified by changing the operational parameters. Therefore, they are proper candidates for adsorption applications including filtration /separation and could be employed as an adsorbent filter for lithium mining process [7].

Many studies have been studied with carbon nanotubes (CNTs) for their utilization in Li ion batteries. CNTs have been used for the anode systems due to their better Li ion adsorption capacities [8-11]. The CNTs are one of the promising materials because of their large surface areas and $\mathrm{Li}$ adsorption properties. The electrostatic interactions between hexagonal sites on carbon nanotube and $\mathrm{Li}^{+}$can enhance the sorption capacity of adsorbent materials [12]. In this study, electrospun fiber mats were fabricated for the lithium sorption from geothermal brines. Solutions of various polymers were subjected to electrospinning and treated with CNT's. PMMA (carbonyl), PAN (nitrile), and chitosan (amine) have functional groups for the metal binding. As-prepared electrospun mats were performed for lithium sorption in neat and CNT treated forms. Three different brine concentrations were used because of high salinity properties of Tuzla geothermal field.

\section{Materials and Methods}

\section{Materials.}

Polyacrylonitrile was synthesized in the laboratory by using acrylonitrile and benzoyl peroxide that purchased from Fluka. Poly(methyl methacrylate) $\left(\mathrm{M}_{v}=350 \mathrm{kDa}\right.$, Sigma Aldrich) and polyacrylonitrile was dissolved in $N, N$-Dimethylformamide (Carlo Erba Reagents Group), Chitosan powder (MMW and 70-75\% degree of deacetylation, Sigma Aldrich) were dissolved in 1,1,1,3,3,3- hexafluoroisopropanol (HFIP) (Sigma Aldrich). Single-walled carbon nanotube [13, 14] (SWCNT, taken from Prof. Dr. Yusuf Selamet group at İzmir Institute of Technology Department of Physics) was used for the modification of fabricated electrospun fiber mats. Before modification, single-walled carbon nanotubes (SWCNT) were purified and functionalized with $\mathrm{NH}_{4} \mathrm{OH}$ (Sigma Aldrich), $\mathrm{H}_{2} \mathrm{SO}_{4}$ (Fluka) and $\mathrm{HCl}$ (Merck). $\mathrm{HNO}_{3}$ (Merck) was used to acidify the brine samples.

Purification of SWCNT. SWCNTs were purified with the following procedure. $0.1 \mathrm{~g} \mathrm{SWCNT}$ was weighed and taken into a beaker. The mixture of $\mathrm{H}_{2} \mathrm{SO}_{4}(5 \mathrm{~mL})$ and $\mathrm{HNO}_{3}(1.7 \mathrm{~mL})$ was poured onto $0.1 \mathrm{~g}$ of SWCNTs and sonicated for $1 \mathrm{~h}$. After sonication, it was held at fume hood for about $12 \mathrm{~h} .1 .7 \mathrm{~mL} \mathrm{HCl}$ was then added to the solution. $19 \mathrm{~mL} \mathrm{NH}_{4} \mathrm{OH}$ solution was slowly poured into the mixture until the end of the gas release. At $\mathrm{pH} \mathrm{7,} \mathrm{the} \mathrm{solution} \mathrm{was} \mathrm{filtered} \mathrm{with} \mathrm{a} 0.2 \mu \mathrm{m}$ cellulose acetate filter paper. The filtrated product was taken and deionized water was added until adjusting the solution's $\mathrm{pH}$ in the range of 5.5- 6.0. That solution was further filtered with a $0.2 \mu \mathrm{m}$ porous filter paper. The final product was dried in a drying oven for 2 hours at $40{ }^{\circ} \mathrm{C}$ [15].

Synthesis of Polymers. PAN synthesis was carried out via solution polymerization. AN (5 g, $0.094 \mathrm{~mol}$ ) was dissolved in DMF $(5 \mathrm{~mL})$. The mixture was purged with $\mathrm{N}_{2}$ to get rid of oxygen in the system. Subsequently, BPO $(114 \mathrm{mg}, 0.047 \mathrm{mmol})$ was added to a system as an initiator. The reaction was maintained at $80{ }^{\circ} \mathrm{C}$ under the $\mathrm{N}_{2}$ atmosphere in the flask that is equipped with a condenser for $12 \mathrm{~h}$. Mixture was precipitated in methanol drop by drop. Then, PAN beads were dried under vacuum for $3 \mathrm{~h}$.

Preparation of Electrospinning Fiber Mats. The different concentrations of polymer solutions were prepared (Table 1). Chitosan $(0.4 \mathrm{wt} \%)$ was dissolved in HFIP [15]. PMMA (5 and 7.5\%) and PAN $(5,15$, and 20\%) were dissolved separately in DMF. 
Chitosan, PAN, and PMMA solutions were subjected to electrospinning using a homemade set-up $[16,17]$. Table 1 shows the working parameters i.e., polymer solution concentration, flow rate, and electrical field.

Table 1. The fabrication conditions of the fiber mats

\begin{tabular}{cccc}
\hline Polymer & $\begin{array}{c}\text { Concentration } \\
(\mathbf{w t} \text { \%) }\end{array}$ & $\begin{array}{c}\text { Flow Rate } \\
(\mathbf{m L} / \mathbf{h})\end{array}$ & $\begin{array}{c}\text { Electrical } \\
\text { Field } \\
(\mathbf{k V} / \mathbf{c m})\end{array}$ \\
\hline Chitosan & 0.4 & 1.0 & 2.1 \\
\hline \multirow{2}{*}{ PAN } & 5.0 & 1.5 & 3.0 \\
& 15 & 2.0 & 2.6 \\
\multirow{2}{*}{ PMMA } & 20 & 2.2 & 2.7 \\
\hline
\end{tabular}

Modification of Electrospun Fiber Mats. The electrospun fiber mats were detached from the aluminum foil. To examine the effect of SWCNT on the lithium sorption, they were treated with water-SWCNT dispersion in a water bath shaker (GFL 1083, Germany) for $3 \mathrm{~h}$. Before modification, SWCNT (1 mg) were powdered well in an agate mortar and dispersed in water $(100 \mathrm{~mL})$ using the ultrasonic bath for $15 \mathrm{~min}$. To determine the surface morphology of the fibers, scanning electron microscopy (SEM) was performed using Carl Zeiss 300 VP and fiber diameters were calculated by ImageJ software.

Sorption Studies. Brine sample was collected from the well head part of the Tuzla power plant into the $1 \mathrm{~L}$ plastic bottle and acidified with $1 \%(\mathrm{v} / \mathrm{v})$ nitric acid to prevent precipitation of the metal hydroxides [18]. Carbon nanotube modified electrospun fiber mats $(\sim 1.5 \mathrm{mg})$ were treated with brine solutions in the ratios of $1 / 1,1 / 10$, and $1 / 100$ for $24 \mathrm{~h}$ in the shaker at room temperature. Sorption experiments were performed by the batch technique and $\mathrm{pH}$ of the solutions was adjusted to 5-6 with $0.01 \mathrm{M} \mathrm{HNO}_{3}$ solution. Determination of the remaining lithium concentration was performed with an atomic emission spectrometer (AES, Thermo Elemental SOLAAR M6, USA).

\section{Result and Discussion}

Chitosan, PMMA, and PAN fibers were fabricated and functionalized with single walled carbon nanotubes (SWCNT) for their potential use in lithium sorption from geothermal brines. Figure 1 shows the micrographs of prepared fibers before and after SWCNT modification. The neat fibers are uniformly distributed and do not show any distinct morphological changes. The chitosan and PAN fibers show nearly identical diameter distributions with an average diameter of $\approx 140 \pm$ $30 \mathrm{~nm}$ (inset figures in (a) and (c)).

Otherwise the PMMA fibers are thicker than those of chitosan and PAN. The diameter varies between 435 and $830 \mathrm{~nm}$ with an average of $\approx 610 \pm 120 \mathrm{~nm}$ (inset figure in (b). After SWCNT modification, it can be observed that obvious bundles and aggregates of carbonaceous particles distributed over the fibers (the right columns of Figure 1) [19, 20]. Hence, the postmodification of the fibers resulted in successful immobilization of SWCNT on the surface of the fibers.

With the aim of increasing the sorption efficiency of chitosan, PMMA, and PAN fibers toward lithium ions, the effect of SWCNT modification was investigated. Lithium sorption experiments were conducted with a dilution factor of 1,10 , and 100 due to the high salinity of the geothermal brine. Figure 2 demonstrates the sorption percentages of $\mathrm{Li}^{+}$ions onto the different fibrous mats in geothermal brines. Not surprisingly, higher sorption values were obtained at a dilution factor of 100 , where the concentration of the analyte is not sufficiently high for the saturation of available functional sites. When considering the neat fibers, the order of increasing sorption percentage at low concentration is PMMA $(26 \%)<$ PAN $(42 \%)<$ Chitosan $(50 \%)$. The 
observed relatively higher uptake in chitosan can be attributed to the free electron pair on amine groups which have the chelating ability for metal ions [16].
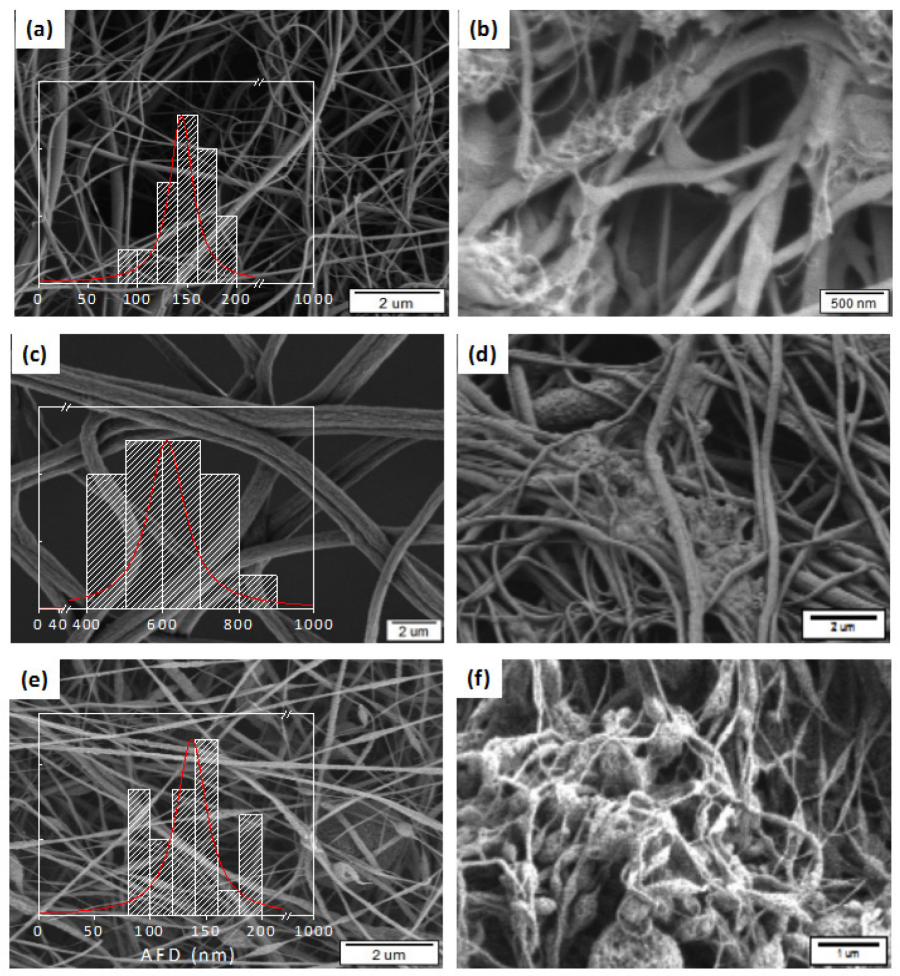

Fig. 1. SEM micrographs of untreated and SWCNT treated fiber mats $(a, b)$ chitosan $(0.4 \mathrm{wt} \%)$, (c, d) PMMA (7.5 wt \%), and (e, f) PAN (15 wt \%)

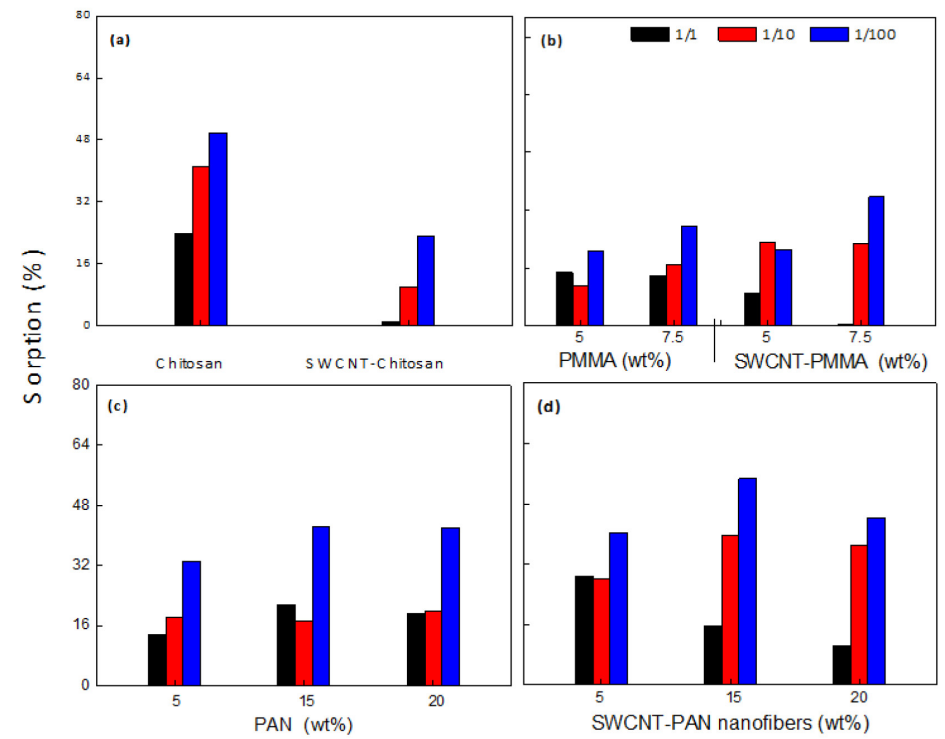

Fig. 2. Sorption efficiencies of neat and SWCNT-modified fiber mats of (a) chitosan $(0.4 \mathrm{wt} \%)$,

(b) PMMA (7.5 wt\%), (c, d) PAN (15 wt\%) at different brine concentrations

The carbonyl group of PMMA and the nitrile group of PAN play a role in sorption process. However, in order to enhance the sorption capacity of these polymers, various additives (such as zeolite, clay minerals) are used [21] or surface functionalization with amidoxime, imidazole, and metal oxides is required [17]. The lower sorption performance of PMMA fibers can also be related to their thicker diameter compared to the fiber of PAN and chitosan. The amount of sorption increases with the decreasing fiber diameter as a result of the larger effective surface area of the fibers [22]. However, SWCNT modification has a negative influence on the $\mathrm{Li}^{+}$ion removal efficiency probably due to the blocking of the amine groups decreasing their interactions with the analyze (Figure 2a). 
On the other hand, SWCNT treatment slightly increases the sorption efficiency of PMMA fibers in terms of increasing the polymer content (Figure 2b). Likewise, the surface functionalization increases the sorption efficiency of PAN fibers almost linearly with the solid content except for $1 / 1$ dilution. Maximum sorption percentage of $55 \%$ was obtained at $1 / 100$ dilution with SWCNT modified PAN (15 wt $\%$ ) fibers (Figure 2c-d). The reason for the enhancement with the modified fibers may be the hexagon sites of SWCNT which are responsible for the $\mathrm{Li}^{+}$sorption [12].

\section{Conclusion}

Lithium mining from geothermal resources has the potential to improve the geothermal economy in terms of increasing geothermal energy production. Electrospun fiber mats of chitosan, PMMA, and PAN were fabricated and functionalized with single-walled carbon nanotubes (SWCNT) for the lithium $\left(\mathrm{Li}^{+}\right)$sorption from geothermal brines. On one hand, SWCNT enhanced the $\mathrm{Li}^{+}$sorption capacity of PMMA and PAN fibers to some extent, on the other hand, reduced the sorption performance of chitosan fibers at low concentrations. The highest sorption percentage of $55 \%$ was obtained at 1/100 dilution with SWCNT modified PAN (15 wt\%) fibers probably due to the hexagon sites of SWCNT which are responsible for the $\mathrm{Li}^{+}$sorption.

\section{Acknowledgement}

The authors are thankful to the research assistant Atike İnce Yardımc1 for her supplement of single walled carbon nanotube. The authors also thanks to The Scientific and Technological Council of Turkey (TUBITAK) for the fund with a project number $114 Z 940$.

\section{References}

[1] X. Xu, Y.M. Chen, P.Y. Wan, K. Gasem, K.Y. Wang, T. He, H. Adidharma, M.H. Fan, Extraction of lithium with functionalized lithium ion-sieves, Progress in Materials Science, 84 (2016) 276-313.

[2] W. Xiang, S.K. Liang, Z.Y. Zhou, W. Qin, W.Y. Fei, Extraction of lithium from salt lake brine containing borate anion and high concentration of magnesium, Hydrometallurgy, 166 (2016) 9-15.

[3] M.M. Demir, A.Baba, V. Atilla, M.. Inanli, Types of the scaling in hyper saline geothermal system in northwest Turkey, Geothermics, 50 (2014) 1-9.

[4] S. Nishihama, K. Onishi, K. Yoshizuka, Selective recovery process of lithium from seawater using integrated ion exchange methods, Solvent Extraction and Ion Exchange, 29 (2011) 421-431.

[5] A. Greiner, J.H. Wendorff, Electrospinning: A fascinating method for the preparation of ultrathin fibres, Angewandte Chemie-International Edition, 46 (2007) 5670-5703.

[6] M.M. Demir, I. Yilgor, E. Yilgor, B. Erman, Electrospinning of polyurethane fibers, Polymer, 43 (2002) 3303-3309.

[7] W.J. Chung, R.E.C. Torrejos, M.J. Park, E.L. Vivas, L.A. Limjuco, C.P. Lawagon, K.J. Parohinog, S.P. Lee, H.K. Shon, H. Kim, G.M. Nisola, Continuous lithium mining from aqueous resources by an adsorbent filter with a 3D polymeric nanofiber network infused with ion sieves, Chemical Engineering Journal, 309 (2017) 49-62.

[8] Y. Chen, X. Li, K. Park, J. Song, J. Hong, L. Zhou, Y.-W. Mai, H. Huang, J.B. Goodenough, Hollow carbon-nanotube/carbon-nanofiber hybrid anodes for Li-ion batteries, Journal of the American Chemical Society, 135 (2013) 16280-16283.

[9] B.J. Landi, M.J. Ganter, C.D. Cress, R.A. DiLeo, R.P. Raffaelle, Carbon nanotubes for lithium ion batteries, Energy \& Environmental Science, 2 (2009) 638-654. 
[10] A.L.M. Reddy, M.M. Shaijumon, S.R. Gowda, P.M. Ajayan, Coaxial mno2/carbon nanotube array electrodes for high-performance lithium batteries, Nano Letters, 9 (2009) 1002-1006.

[11] H. Xia, M. Lai, L. Lu, Nanoflaky MnO2/carbon nanotube nanocomposites as anode materials for lithium-ion batteries, Journal of Materials Chemistry, 20 (2010) 6896-6902.

[12] W. Koh, J.I. Choi, S.G. Lee, W.R. Lee, S.S. Jang, First-principles study of Li adsorption in a carbon nanotube-fullerene hybrid system, Carbon, 49 (2011) 286-293.

[13] A. Rashidi, M. Akbarnejad, A. Khodadadi, Y. Mortazavi, A. Ahmadpourd, Single-wall carbon nanotubes synthesized using organic additives to co-mo catalysts supported on nanoporous mgo, Nanotechnology, 18 (2007) 315605.

[14] A.I. Yardimci, S. Yilmaz, Y. Selamet, The effects of catalyst pretreatment, growth atmosphere and temperature on carbon nanotube synthesis using co-mo/mgo catalyst, Diamond and Related Materials, 60 (2015) 81-86.

[15] M. Yücel, Fabrication of thin layer polymer-based biointerphase for biosensing application, in İzmir Institute of Technology, 2017.

[16] N. Horzum, E. Boyac1, A.E. Eroglu, T. Shahwan, M.M. Demir, Sorption efficiency of chitosan nanofibers toward metal ions at low concentrations, Biomacromolecules, 11 (2010) 3301-3308.

[17] N. Horzum, T. Shahwan, O. Parlak, M.M. Demir, Synthesis of amidoximated polyacrylonitrile fibers and its application for sorption of aqueous uranyl ions under continuous flow, Chemical Engineering Journal, 213 (2012) 41-49.

[18] A. Celik, G. Koc, E. Erdogan, T. Shahwan, A. Baba, M.M. Demir, Use of electrospun fiber mats for the remediation of hypersaline geothermal brine, Desalination and Water Treatment, 62 (2017) 94-100.

[19] H. Hu, B. Zhao, M.E. Itkis, R.C. Haddon, Nitric acid purification of single-walled carbon nanotubes, The Journal of Physical Chemistry B, 107 (2003) 13838-13842.

[20] J.-M. Moon, K.H. An, Y.H. Lee, Y.S. Park, D.J. Bae, G.-S. Park, High-yield purification process of singlewalled carbon nanotubes, The Journal of Physical Chemistry B, 105 (2001) 56775681.

[21] J.J.L. Lee, B.C. Ang, A. Andriyana, M.I. Shariful, M. Amalina, Fabrication of PMMA/zeolite nanofibrous membrane through electrospinning and its adsorption behavior, Journal of Applied Polymer Science, 134 (2017).

[22] T. Isık, N. Horzum, Ü.H. Yildı, B. Liedberg, M.M. Demir, Utilization of electrospun polystyrene membranes as a preliminary step for rapid diagnosis, Macromolecular Materials and Engineering, 301 (2016) 827-835. 